\title{
The University of Tokyo Atacama Observatory 6.5m telescope: project overview and current status
}

M. Doi, T. Miyata, Y. Yoshii, K. Kohno, M. Tanaka, et al.

M. Doi, T. Miyata, Y. Yoshii, K. Kohno, M. Tanaka, K. Motohara, T. Minezaki, K. Kawara, S. Sako, T. Morokuma, Y. Tamura, T. Tanabe, B. Hatsukade, H. Takahashi, M. Konishi, T. Kamizuka, N. Kato, T. Aoki, T. Soyano, K. Tarusawa, T. Handa, S. Koshida, L. Bronfman, M. T. Ruiz, M. Hamuy, R. Mendez, G. Garay, A. Escala, "The University of Tokyo Atacama Observatory 6.5m telescope: project overview and current status," Proc. SPIE 10700, Groundbased and Airborne Telescopes VII, 107000W (6 July 2018); doi: $10.1117 / 12.2313099$

Event: SPIE Astronomical Telescopes + Instrumentation, 2018, Austin, Texas, United States 


\title{
The University of Tokyo Atacama Observatory 6.5m telescope: Project overview and current status
}

\author{
Doi, M. ${ }^{\mathrm{a}, \mathrm{b}}$, Miyata, T. ${ }^{\mathrm{a}}$, Yoshii, Y.., d, Kohno, K. ${ }^{\mathrm{a}, \mathrm{b}}$, Tanaka, M. ${ }^{\mathrm{a}}$, Motohara, K. ${ }^{\mathrm{a}}$, Minezaki, T. ${ }^{\mathrm{a}}$, \\ Kawara, K. ${ }^{a}$, Sako, S. ${ }^{a}$, Morokuma T. ${ }^{\text {, }}$, Tamura, Y. ${ }^{\mathrm{a}}$, Tanabe T. ${ }^{\mathrm{a}}$,Hatsukade B. ${ }^{\mathrm{a}}$, Takahashi, H. ${ }^{\mathrm{a}}$, \\ Konishi M. ${ }^{a}$, Kamizuka, T. ${ }^{a}$,Kato, N. ${ }^{a}$,Aoki, T. ${ }^{c}$, Soyano T. ${ }^{\mathrm{c}}$, Tarusawa, K. ${ }^{\mathrm{c}}$, Handa T. ${ }^{\mathrm{e}}$, Koshida \\ S. ${ }^{\text {, }}$ Bronfman, L. ${ }^{\mathrm{g}}$, Ruiz, M. T. ${ }^{\mathrm{g}}$, Hamuy M. ${ }^{\mathrm{g}}$, Mendez R..$^{\mathrm{g}}$, Garay, G. ${ }^{\mathrm{g}}$, Escala, A. ${ }^{\mathrm{g}}$

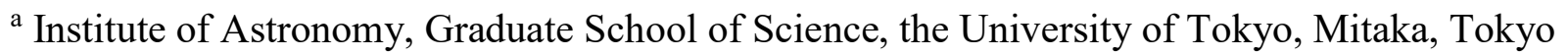 \\ 181-0015, Japan; \\ ${ }^{\mathrm{b}}$ Research Center for the Early Universe, Graduate School of Science, The University of Tokyo, \\ Bunkyo-ku, Tokyo 113-0033, Japan; \\ ${ }^{c}$ Kiso Observatory, Institute of Astronomy, Graduate School of Science, the University of Tokyo, \\ Kiso-machi, Nagano 397-0101, Japan; \\ ${ }^{\mathrm{d}}$ Steward Observatory, University of Arizona, 933 North Cherry Avenue, Rm. N204, Tucson, AZ \\ 85721-0065, USA; \\ e Department of Physics and Astronomy, Kagoshima University, Korimoto, Kagoshima 890-0065, \\ Japan;

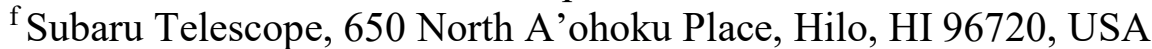 \\ g. Department of Astronomy, University of Chile, Santiago, Chile
}

\begin{abstract}
The University of Tokyo Atacama Observatory Project is to construct and operate a $6.5 \mathrm{~m}$ infrared telescope at the summit of Co. Chajnantor (5640m altitude) in northern Chile, promoted by the Institute of Astronomy of the University of Tokyo. Thanks to the dry climate $(\mathrm{PWV} \sim 0.5 \mathrm{~mm})$ and the high altitude, excellent observation condition in the NIR to MIR wavelengths is achieved. The telescope has two Nasmyth foci where two facility instruments, SWIMS for the near-infrared and MIMIZUKU for the mid-infrared, are installed and two foldedCassegrain foci for carry-in instruments. All these four foci can be switched by rotating a tertiary mirror. The final focal ratio is 12.2 and the foci have large field-of-view of 25 arcmin in diameter.

We adopted a $6.5-\mathrm{m} \mathrm{F} / 1.25$ light-weighted borosilicate honeycomb primary mirror and its support system that are developed by Steward Observatory Richard F. Caris Mirror Lab. An enclosure has the shape of carousel, and large ventilation windows with shutters control the wind to flush heat inside the enclosure. A support building with a control room, a mirror coating system and maintenance facilities is located at the side of the enclosure. The mirror coating system consists of a large aluminizing chamber and a mirror washing facility. The operation of the telescope will be remotely carried out from a base facility at San Pedro de Atacama, 50km away from the summit. Development of the two facility instruments has already been completed and they are transported to Hilo, Hawaii in 2017. We are going to carry out engineering observations of those instruments on the Subaru telescope for clearing up technical issues and verifying their performance. The existing summit access road from the ALMA concession area was laid in 2006, however, it is too narrow to carry large components of the telescope and the ancillary facilities such as the primary mirror, its cell, and the aluminizing chamber. The road is being expanded so that it has the width of $>5 \mathrm{~m}$ for straight portion and $>7 \mathrm{~m}$ for curved portion.. The telescope mount and the enclosure are being pre-assembled for functional and performance tests in Japan. All telescope system will be assembled at the summit and see the engineering first light early 2019.
\end{abstract}

Keywords: TAO, telescope project, infrared, Atacama, high altitude

Ground-based and Airborne Telescopes VII, edited by Heather K. Marshall,

Jason Spyromilio, Proc. of SPIE Vol. 10700, 107000W · C 2018 SPIE

CCC code: $0277-786 \mathrm{X} / 18 / \$ 18 \cdot$ doi: $10.1117 / 12.2313099$ 


\section{INTRODUCTION}

Institute of Astronomy, the University of Tokyo has been promoting a project to install a $6.5 \mathrm{~m}$ infrared-optimized telescope at the summit of Co. Chajnantor in northern Chile, called the University of Tokyo Atacama Observatory (TAO) project ${ }^{[1][2][3][4]}$ (P.I. Yuzuru Yoshii). Thanks to the extreme altitude of the site $(5640 \mathrm{~m})$, the perceptible water vapor at the site is as low as $<0.5 \mathrm{~mm}^{[5][6][7]}$ and highly transparent atmosphere becomes available in the infrared wavelength ${ }^{[7]}$.

Following the success of a pathfinder $1.0 \mathrm{~m}$ telescope "miniTAO" installed at the site in $2009^{[7][8][9][10][11]}$, we started the development of the $1^{\text {st }}$ generation instruments SWIMS and MIMIZUKU in 2010. Both are almost completed and engineering observations at the Subaru telescope are now underway, Fabrication of the telescope structure started in 2012, and its preassembly in Japan has completed on March 2018. Finally, on November 2017, the first stone ceremony was held and on-site construction activities have started. The telescope is now scheduled to see the engineering first light in 2019 .

In this paper, we report the current status of the project, mainly concentrate to the update from the previous report ${ }^{[4]}$.

\section{TELESCOPE}

\subsection{Optics}

A Ritchey-Chretien optical configuration is adopted for the TAO $6.5 \mathrm{~m}$ telescope. The sizes and shapes of the primary, secondary, and tertiary mirrors are $6.5 \mathrm{~m}$ (circular, convex, F/1.25), $0.9 \mathrm{~m}$ (circular, concave), and $1.1 \mathrm{~m} \mathrm{x} 0.75 \mathrm{~m}$ (elliptical, flat), respectively. All these three mirrors are made of borosilicate and have light-weighted honeycomb structure. The telescope is optimized for infrared observations and the undersize secondary mirror is adopted. Then, the effective diameter of the primary mirror is reduced to be $6153.8 \mathrm{~mm}$ in diameter. The final focal ratios at the Nasmyth and folded-Cassegrain foci are 12.2, which is the same as that of the Cassegrain focus of the Subaru 8.2m telescope to share observing instruments without significant modification. The unvignetted field-of-view is 25 arcmin in diameter and the plate scale is and $2.75 \mathrm{\prime \prime} / \mathrm{mm}$. A diameter of $80 \%$ encircled energy $(\theta 80)$ is set to be 0.32 " at a wavelength of $\lambda=500$ $\mathrm{nm}$ according to the error budget for the telescope image quality ${ }^{[12]}$.

The primary mirror is supported and controlled actively by pneumatic actuators and this concept of the support system is followed by those of Magellan telescopes, MMT, and Large Binocular Telescope. This function is achieved by realtime wavefront measurements, which are described in Section 2.3. Functional tests of the primary mirror cell with the pneumatic actuators, hardpoint actuators, and static supports have been almost completed. Measurement of influence functions of the actuators will be carried out in this summer with using the real primary mirror.

\subsection{Telescope Mount}

The telescope mount is a tripod-disk type alt-azimuth mount with hydrostatic bearings and friction drives, in a similar way as the Magellan telescopes and the Large Binocular Telescope. Two Nasmyth ports are provided for the large facility instruments and two auxiliary folded-Cassegrain ports are prepared for carry-in instruments on the center section. The tertiary mirror switches the incident beam to select an instrument mounted on those focal ports, which will enable us to use different instruments at the same night easily.

The telescope mount is designed and fabricated by Nishimura Co. Ltd. Figure 2 shows the telescope mount assembled in Japan for the functional and performance tests. The integration tests of equipments to the telescope mount such as the instrument cart and the mass simulator of the facility instrument were also performed. After the shop assembly, the telescope mount was disassembled and packed up for shipping to Chile. 


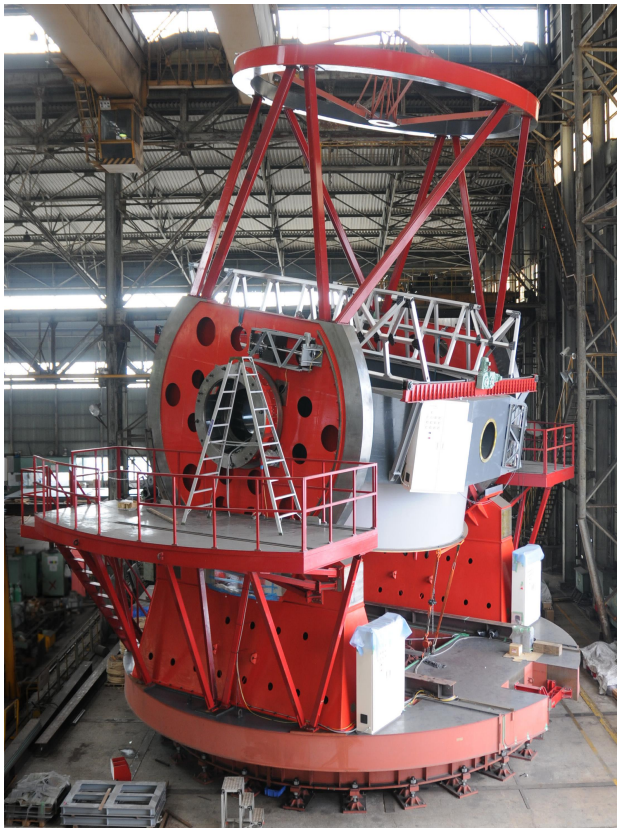

Figure 1: Telescope mount temporarily assembled in Japan

\subsection{Guider and Wavefront Sensors}

The flexure of the telescope truss results in misalignment of the primary and secondary mirrors. To keep their good collimation and focus, the secondary mirror will be shifted and tilted by a hexapod-type mount fabricated by PI-Japan Co., Ltd. The amount of correction will be approximately determined by the elevation angle and the truss temperature.

A wavefront sensor will be installed on the each telescope focus, which will routinely measure the wavefront error of the telescope optics during the observation to make fine correction of the alignment of the secondary mirror as well as the surface figure of the primary mirror. The integrated auto-guider and wavefront-sensor system is designed and fabricated by LLP Kyoto-Nijikobou Co. Ltd. It has two probes mounted on r-theta stages; one is for a conventional offset guider, and the other is for a Shack-Hartmann sensor. The system was temporarily mounted in the instrument derotator of the telescope and examined during the shop assembly. 


\section{INSTRUMENTS}

\subsection{Near-Infrared Imager and Multi-Object Spectrograph: SWIMS}

SWIMS, a Simultaneous-color Wide-field Infrared Multi-object Spectrograph, covers the NIR wavelength from 0.9 to 2.5 micron, and capable of either imaging or multi-object spectroscopy with wide field-of-view of 9.6 arcmin diameter with a pixel scale of 0.126 arcsec/pixel. It has two optical arms by inserting a dichroic mirror in the collimated beam: one covering 0.9-1.45 um and the other 1.45-2.5 um. Combined with the almost continuous atmospheric windows at the site, we can obtain multi-object spectra with spectral resolution of $\lambda / \Delta \lambda \sim 1000$ in the full range of the NIR wavelength in a single exposure.

After passing several reviews by the Subaru review committee, SWIMS has been transported to Hawaii in August 2017 to carry out first-light and commissioning observations on the Subaru Telescope, prior to the completion of the TAO $6.5 \mathrm{~m}$ telescope. The commissioning observations, which is mainly focused on the evaluation of the imaging performance, are allocated from the end of May for 2.5 nights in total. The evaluation of the MOS performance is being proposed to the next semester.

After the completion of the TAO $6.5-\mathrm{m}$ telescope, the instrument will be transported to Chile and will see the scientific first light in FY2020. Detail information is described in an accompanying paper by Konishi et al. $(2018)^{[13]}$

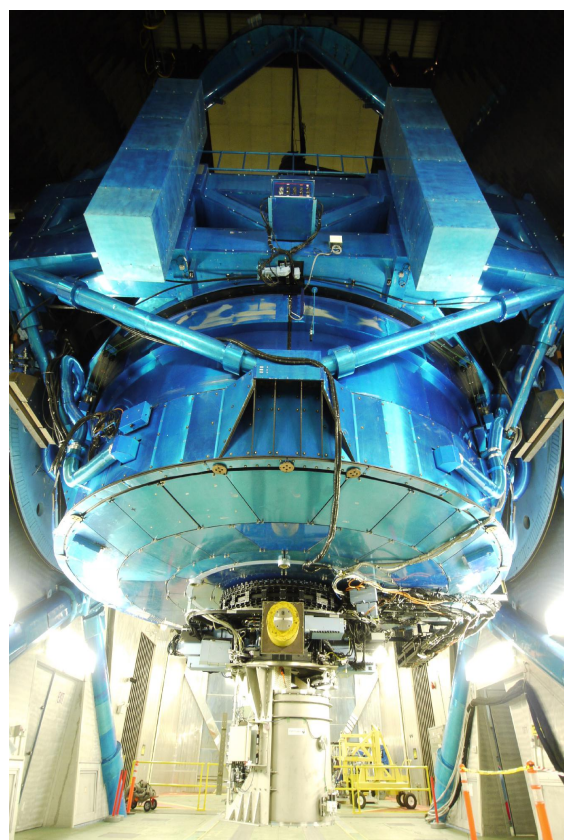

Figure 2: Near-infrared instrument SWIMS installed on the Cassegrain focus of the Subaru Telescope for its commissioning observations.

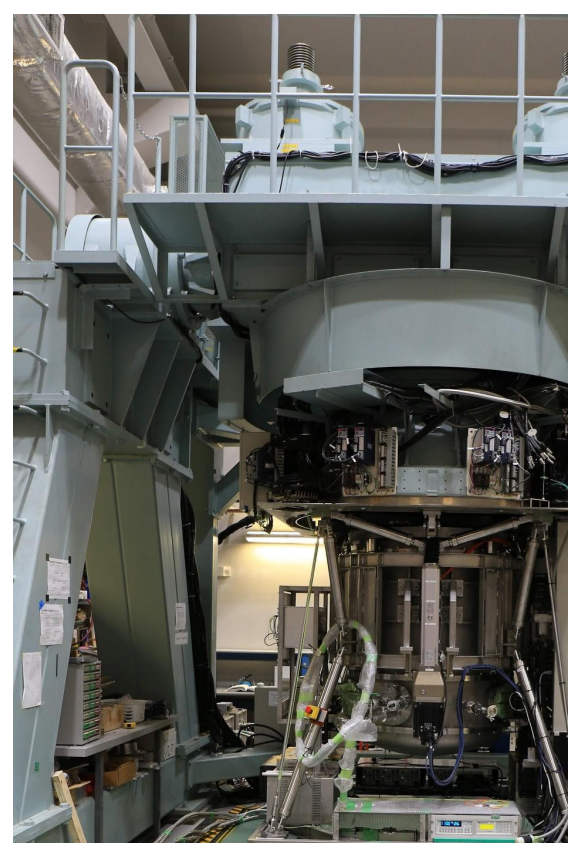

Figure 3: Mid-infrared instrument MIMIZUKU attaching on the telescope simulator in the Hilo base facility of NAOJ 


\subsection{Mid-Infrared Imager and Spectrograph: MIMIZUKU}

Mid-Infrared Multi-field Imager for gaZing at the UnKnown Universe (MIMIZUKU) is a mid-infrared camera and spectrograph developed as the $1^{\text {st }}$-generation mid-infrared instrument for the TAO $6.5-\mathrm{m}$ telescope $\mathrm{e}^{[14][15][16][17]}$. It has capabilities of imaging and low-resolution $(\mathrm{R}<620)$ spectroscopy in a wide wavelength range from 2 to 38 microns with three optical channels, called NIR, MIR-S, and MIR-L. Their covering wavelengths are 2-5.3, 6.8-26, and 24-38 microns, respectively. Only one channel can be used at once, however, it can be switched within a minute. Therefore, we can observe light in this wide wavelength range at almost the same time without time-consuming instrument exchange. In addition to this unique capability, MIMIZUKU has a special optical device called Field Stacker ${ }^{[24]}$. This enables us to carry out simultaneous observations of two objects within 25 arcmin, usually impossible to cover with one detector field. This capability is used for simultaneous observations of target and calibration objects. Such observation technique is effective for accurate calibration of the atmospheric transmittance because it can accurately compensate the temporal change of atmospheric transmittance, and it is expected that we can improve photometric accuracy to the order of one percent even in the wavelength regions with low atmospheric transmittance. This capability is also useful for spectroscopic observations in such low-transmittance bands like 2.7- and 20-micron bands ${ }^{[17]}$. These possibilities enable us to perform long-term infrared monitoring and obtain good quality spectroscopic data even from the ground. We aim to study time-varying events which occur around young stellar objects, evolved stars, novae, supernovae, and so on for understanding the formation, destruction, and alteration of dust grains in such objects.

The development of MIMIZUKU started in 2010, and its progresses has been reported in the past conferences ${ }^{[14][15][16][17][24][25]}$. Currently, the MIR-S channel is completed, although the other channels are still under development. In order to evaluate the on-sky performance of MIMIZUKU and effectivity of the Field Stacker device on calibration, we proposed engineering observations on the Subaru telescope because TAO is still under construction. Our proposal is kindly approved, and MIMIZUKU has been transferred to Hawaii in October 2017. After the transportation, MIMIZUKU has been checked for its functions, and no problem was found. The test results and related studies are reported in this conference ${ }^{[25][25][25]}$. Our first on-sky observation is planned on July 2-4, 2018. In this observation. We will check basic observation functions and mainly evaluate imaging capabilities with and without Field Stacker. After another engineering run in 2018, which has been already proposed, MIMIZUKU will be transported to TAO. Full science operations of MIMIZUKU on the TAO telescope are planned to start in 2019 or later.

\section{3 $2^{\text {nd }}$ Generation Instruments}

In addition to the facility instruments above, we have two 2nd generation instrument plans installed on the folded Cassegrain foci.

One is a Y-band high-resolution spectrograph, called TARdYS (TAO AIUC high Resolution (d) Y-band Spectrograph ${ }^{[25]}$, being developed at Centro de Astro-Ingenieria de la Universidad de Catolica (AIUC). It is a fiber-fed, $\mathrm{R}=60,000$ high resolution infrared spectrograph covering $0.843-1.117 \mu \mathrm{m}$. Most of optical components except for the final lens of a camera optics are kept under room temperature to keep the size and complexity of the spectrograph as small as possible. The main science target is radial-velocity (RV) measurement of M-dwarf stars to discover exoplanets, and RV measurement precision of $2.2 \mathrm{~m} / \mathrm{s}$ is expected in combination of a laser comb system.

The other is an optical imager / spectrograph optimized for shorter wavelength, now under conceptual design phase. It is expected to have an imaging mode with $\sim 30$ filters, and a long-slit spectroscopy mode with $\mathrm{R}=1000$. Wavelength coverage is $300-900 \mathrm{~nm}$ and the optics design is optimized at $<400 \mathrm{~nm}$. This is because atmospheric scattering also becomes smaller than at other site, and better atmospheric transmittance is expected $(\sim 65 \%$ at $340 \mathrm{~nm}$, which is $\sim 10 \%$ better than that of $2500 \mathrm{~m}$ sites). 


\section{FACILITY}

\subsection{Enclosure and Summit Facility}

The TAO summit facility consists of the telescope enclosure with a $\mathrm{d}=24-\mathrm{m}$ and $\mathrm{h}=26-\mathrm{m}$ size and the operation building with a $27-\mathrm{m} \times 17-\mathrm{m} \mathrm{h} \mathrm{h}=14-\mathrm{m}$ size. The observation floor of the enclosure is connected to the second floor of the operation building by a bridge structure. The enclosure has a fixed base structure and a rotating carousel structure with 14 ventilation windows and an overhead crane with a 5-ton capacity. The operation building contains an observation room, a server room, a mirror-coating system, maintenance rooms for observation instruments, and an emergency evacuation room. In the initial operation phase, power supply is generated on the site by three units of power generators with a $350 \mathrm{kVA}$ capacity. An oil and air compressors and a chiller system are installed on the ground floor in the enclosure building. Warm air produced by these heavy instruments and the telescope is exhausted to the far north and south areas of the summit facility through ducts. The power consumption of the summit facility is estimated to be about $140 \mathrm{~kW}$ on average and about $300 \mathrm{~kW}$ at maximum during normal operations and mirror coating works, respectively. A concrete pier of the telescope has a diameter of $10-\mathrm{m}$ and a height of $3.4-\mathrm{m}$ from the ground level. It is isolated from structure of the enclosure and the support buildings

Most of steel parts of the carousel structure have been already fabricated in Japan. In 2016 and 2017, the steel parts and mechanical parts including bogie carts have been pre-assembled in Japan to achieve trouble-free construction at the site of 5,640-m altitude. After performance tests, all components are reassembled and packed to be transported to Chile.

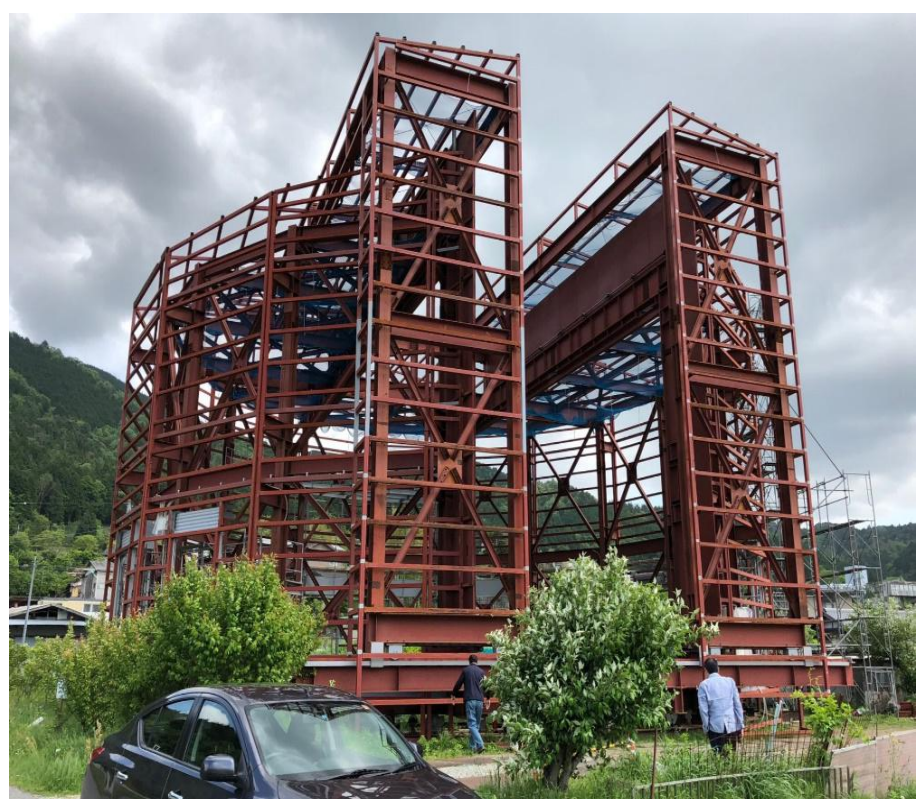

Figure 4: Picture of the carousel structure of the enclosure building pre-assembled in Osawa, Japan. 


\subsection{Mirror Coating System}

In order to fabricate the reflecting film initially on the mirror surface and to maintain its optical performance over a long period, we have a mirror-coating facility being installed at the summit ${ }^{[24]}{ }^{[25]}$. The facility consists of a clean booth for stripping off the old film, a mirror coating chamber, and a cart with a lifter for handling the primary mirror. A conventional evaporation system with a metal pre-wetted filament array is adopted for achieving various optical requests. Among the many development items, the fabrication of the transportation and lifting cart has been already completed (Figure 5). It has efficient performance in load capacity ( $>60$ tons) and maximum lifting height $(1,750 \mathrm{~mm})$. This cart will be also used for installing and removing the primary mirror cell at the telescope assembly phase. A cleaning machine having injection nozzles that can realize an efficient and safe cleaning sequence has also been completed. A test of the evaporation system using dedicated filaments and filament boxes, which are customized to the TAO's requirements, has shown a uniform coating on a test mirror. Figure 6 shows the layout of the mirror coating facility in the summit operation building. All system is arranged so that the operation proceeds smoothly and functionally. Almost all system except for the main chamber have been completed.

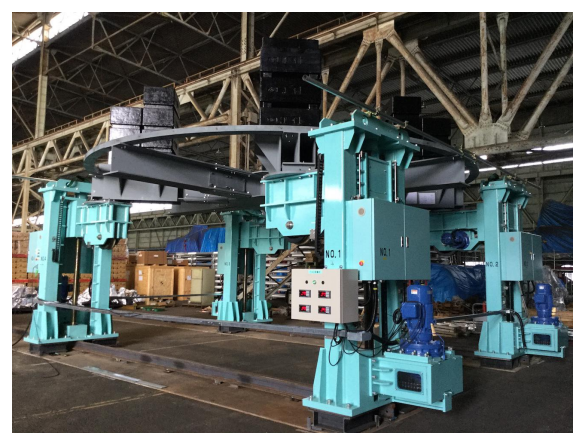

Figure 5: The picture of load and moving test of transportation cart (lifting cart) with weight corresponding to PMC. The alternative stage is used instead of lower chamber.

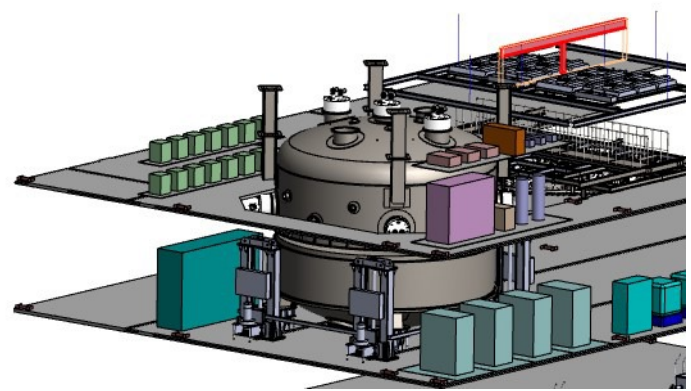

Figure 6 : The layout of mirror coating facility including mirror cleaning system in observation support building. Upper chamber is set on the ceiling of support building. Lower chamber with PMC moves to under the upper chamber by transportation cart on the rail extending from dome enclosure. Pumping units and control units are set on 3rd floor and 2nd floor respectively considering to functionality. Water units are installed on ground floor for safety. 


\subsection{Access Road}

An original access road from the ALMA region ( 5,000 m.a.s.l) to the summit (5640m m.a.s.l) was constructed in 2006 . It had a length of $6 \mathrm{~km}$ and a width of $\sim 3 \mathrm{~m}$. It was enough for the site assessment and the installation of the miniTAO telescope, but too narrow and too steep to bring up components of the enclosure and the TAO telescope. A road expansion plan has been made with consideration of transportation of large components such as the primary mirror (7-m width including a transportation box), the primary mirror cell (7-m diameter), and the coating chamber (upper and lower, both have $7-\mathrm{m}$ diameter). The width is expanded to $6 \mathrm{~m}$, and the maximum slope is set to be $0 \%$ and $12 \%$ at linear and curved portions, respectively. Expansion work is now in progress at the site, and will finish around July 2018.

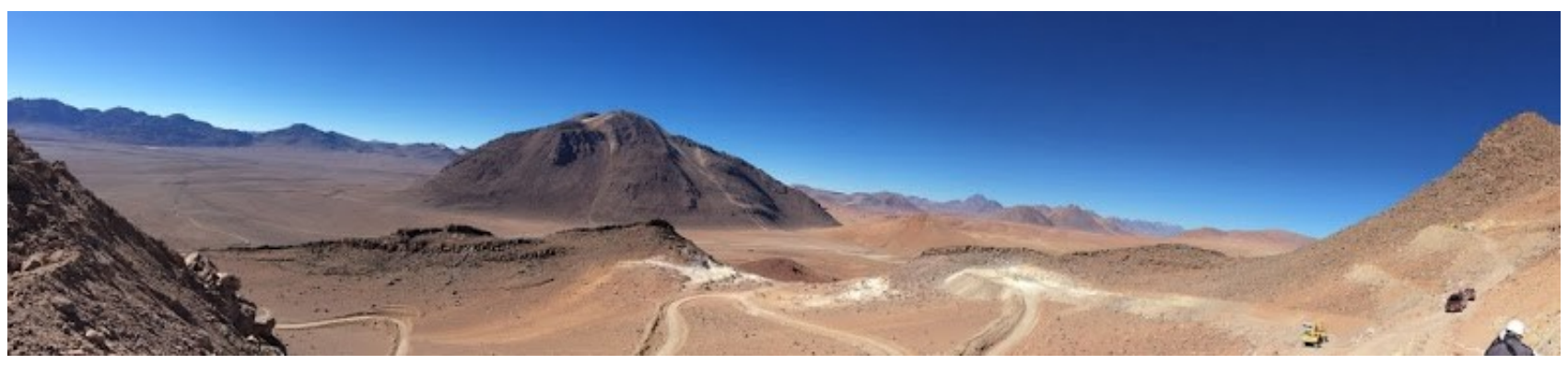

Figure 7: A panoramic photo of the summit access road under expansion work

\section{SCHEDULE}

The TAO project is now in the final stage of the fabrication and construction phase. Major components such as the telescope mount, the primary mirror, and the active mirror system have been successfully completed and carried out final performance verification tests. The transportation plan from Japan/USA to the summit has been also prepared. The summit leveling will be completed in August in 2018, and the construction of the enclosure will start subsequently. We plan to see the first light at early 2019 and start science observations within the FY2019 for the fastest case. 


\section{ACKNOWLEDGEMENT}

This work has been supported by the Grant-in-Aid for Scientific Research (21018003, 21684006, 22253002, 23540261, 24103003, 24244015, 2611460, 25247017, 15K17599, 15H02062) from the JSPS. Part of this work has been supported by Optical \& Near-Infrared Astronomy Inter-University Cooperation Program by the MEXT of Japan, Institutional program for young researcher overseas visits by the JSPS, and NAOJ research grant for universities. Part of this work is also supported by the Advanced Technology Center, NAOJ

\section{REFERENCES}

[1] Yoshii, Y., Doi, M., Handa, T., Kawara, K., Kohno, K., Minezaki, T., Mitsuda, K., Miyata, T., Motohara, K., Tanaka, M., "Tokyo Atacama Observatory Project", Proceedings of the IAU 8th Asian-Pacific Regional Meeting, 35 (2002)

[2] Yoshii, Y., Aoki, T., Doi, M., Handa, T., Kawara, K., Kato, D., Kohno, K., Konishi, M., Koshida, S., Minezaki, T., Mitani, N., Miyata, T., Motohara, K., Sako, S., Soyano, T., Tanabe, T., Tanaka, M., Tarusawa, K., Bronfman, L., Ruiz, M. T., Hamuy, M. "The University of Tokyo Atacama Observatory $6.5 \mathrm{~m}$ telescope project", Proc. SPIE 7733, 773308 (2010)

[3] Yoshii, Y., Doi, M., Kohno, K., Miyata, T., Motohara, K., Kawara, K., Tanaka, M., Minezaki, T., Sako, S., Morokuma, T., Tamura, Y., Tanabe, T., Takahashi, H., Konishi, M., Kamizuka, T., Koshida, S., Kato, N., Aoki, T., Soyano, T., Tarusawa, K., Handa, T., Bronfman, L., Ruiz, M. T., Hamuy, M., Mendez' R., "Overview of University of Tokyo Atacama Observatory 6.5m telescope project", Proc. SPIE 9145, 914507 (2014)

[4] Yoshii, Y., Doi, M., Kohno, K., Miyata, T., Motohara, K., Kawara, K., Tanaka, M., Minezaki, T., Sako, S., Morokuma, T., Tamura, Y., Tanabe, T., Takahashi, H., Konishi, M., Kamizuka, T., Kato, N., Aoki, T., Soyano, T., Tarusawa, K., Handa, T., Koshida, S., Bronfman, L., Ruiz, M. T., Hamuy, M., Garay' G., "The University of Tokyo Atacama Observatory 6.5m telescope: Project overview and current status", Proc. SPIE 9906, 99060R (2016)

[5] Erasmus, F. S. and van Staden, C. A., "A satellite survey of cloud cover and water vapor in northern Chile", A study conducted for Cerro Tololo Interamerican Observatory and University of Tokyo (2001).

[6] Motohara, K., T. Aoki, K. Asano, M. Doi, T. Handa, K. Kawara, D. Kato, N. Kato, K. Kohno, M. Konishi, S. Koshida, T. Minezaki, T. Miyata, T. Nakamura, S. Sako, T. Soyano, T. Tanabe, M. Tanaka, K. Tateuchi, K. Tarusawa, M. Uchiyama, Y. Yoshii, L. Bronfman, M. T. Ruiz, and M. Hamuy, "Site Characteristics of the Summit of Cerro Chajnantor at the 5640 m Altitude", RMxAC 41, 83-86 (2011)

[7] Konishi, M., K. Motohara, K. Tateuchi, H. Takahashi, Y. Kitagawa, N. Kato, S. Sako, Y. K. Uchimoto, K. Toshikawa, R. Ohsawa, T. Yamamuro, K. Asano, Y. Ita, T. Kamizuka, S. Komugi, S. Koshida, S. Manabe, N. Matsunaga, T. Minezaki, T. Morokuma, A. Nakashima, T. Takagi, T. Tanabe, M. Uchiyama, T. Aoki, M. Doi, T. Handa, D. Kato, K. Kawara, K. Kohno, T. Miyata, T. Nakamura, K. Okada, T. Soyano, Y. Tamura, M. Tanaka, K. Tarusawa, Y. Yoshii, "ANIR : Atacama Near-Infrared Camera for the 1.0-m miniTAO Telescope", PASJ 67, 4 (2015)

[8] Minezaki, T., Kato, D., Sako, S., Konishi, M., Koshida, S., Mitani, N., Aoki, T., Doi, M., Handa, T., Ita, Y., Kawara, K., Kohno, K., Miyata, T., Motohara, K., Soyano, T., Tanabe, T., Tanaka, M., Tarusawa, K., Yoshii, Y., Bronfman, L., Ruiz, M. T., Hamuy, M., "The University of Tokyo Atacama 1.0-m Telescope", Proc. SPIE, 773356-1 (2010)

[9] Motohara, K., Konishi, M., Toshikawa, K., Mitani, N., Sako, S., Uchimoto, K. Y., Yamamuro, T., Minezaki, T., Tanabe, T., Miyata, T., Koshida, S., Kato, D., Ohsawa, R., Nakamura, T., Asano, K., Yoshii, Y., Doi, M., Kohno, K., Tanaka, M., Kawara, K., Handa, T., Aoki, T., Soyano, T., Tarusawa, K., Ita, Y., "First Paschen $\alpha$ imaging from the ground: the first light of Atacama Near-Infrared camera on the miniTAO $1 \mathrm{~m}$ telescope", Proc. SPIE, 77353K-1 (2010)

[10] Nakamura, T., Miyata, T., Sako, S., Asano, K., Uchiyama, M., Tanabe, T., Yoneda, M., Ita, Y., Onaka, T., Kataza, H., Aoki, T., Doi, M., Handa, T., Kato, D., Kawara, K., Kohno, K., Konishi, M., Koshida, S., Minezaki, T., Mitani, N., Motohara, K., Ohsawa, R., Soyano, T., Tanaka, M., Tarusawa, K., Toshikawa, K., Yoshii, Y., "MiniTAO/MAX38 first light: 30-micron band observations from the ground-based telescope", Proc. SPIE, 773561-1 (2010) 
[11] Miyata, T., Sako, S., Kamizuka, T., Nakamura, T., Asano, K., Uchiyama, M., Konishi, M., Yoneda, M., Takato, N., Yoshii, Y., Doi, M., Kohno, K., Kawara, K., Tanaka, M., Motohara, K., Minezaki, T., Tanabe, T., Morokuma, T., Tamura, Y., Aoki, T., Soyano, T., Tarusawa, K., Takahashi, H., Koshida, S.., and Kato, N. M., "Evaluations of new atmospheric windows at thirty micron wavelengths for astronomy", Proc. SPIE, 8444, 84446B (2012)

[12] Morokuma, T., Aoki, T., Doi, M., Handa, T., Kamizuka, T., Kato, N., Kawara, K., Kohno, K., Konishi, M., Koshida, S., Minezaki, T., Miyata, T., Motohara, K., Sako, S., Soyano, T., Takahashi, H., Tamura, Y., Tanabe, T., Tanaka, M., Tarusawa, K., and Yoshii, Y., "Design and development status of the University of Tokyo Atacama Observatory 6.5m telescope", Proc. SPIE, 9145, 91453C (2014)

[13] Konishi, M., Motohara, K., Takahashi, H., Kato, N. M., Terao, Y., Ohashi, H., Kono, Y., Kushibiki, K., Tateuchi, K., Kitagawa, Y., Kobayakawa, Y., Todo, S., Aoki, T., Doi, M., Hatsukade, B., Kamizuka, T., Kohno, K., Minezaki, T., Miyata, T., Morokuma, T., Sako, S., Soyano, T., Tanabe, T., Tanaka, M., Tarusawa, K., Tamura, Y., Koshida, S., Ohsawa, R., Uchiyama, M. S., Mori, T., Yamaguchi, J., Yoshida, Y., and Yoshii, Y., "Development status of the simultaneous two-color near-infrared multi-object spectrograph SWIMS for the TAO 6.5m telescope," Proc. SPIE, in this conference

[14] Miyata, T., Sako, S., Nakamura, T., Asano, K., Uchiyama, M., Onaka, T., Sakon, I., Kataza, H., Ita, Y., Aoki, T., Doi, M., Handa, T., Kato, D., Kawara, K., Kohno, K., Konishi, M., Koshida, S., Minezaki, T., Mitani, N., Motohara, K., Soyano, T., Tanabe, T., Tanaka, M., Tarusawa, K., and Yoshii, Y., "Development of a new midinfrared instrument for the TAO 6.5-m Telescope", Proc. SPIE, 7735, 77353P (2010)

[15] Kamizuka, T., Miyata, T., Sako, S., Nakamura, T., Asano, K., Uchiyama, M., Okada, K., Onaka, T., Sakon, I., Kataza, H., Sarugaku, Y., Yoshii, Y., Doi, M., Kohno, K., Kawara, K., Tanaka, M., Motohara, K., Tanabe, T., Minezaki, T., Morokuma, T., Tamura, Y., Aoki, T., Soyano, T., Tarusawa, K., Kato, N., Konishi, M., Takahashi, H., Koshida, S., Tateuchi, K., and Handa, T., "Development of MIMIZUKU: a mid-infrared multi-field imager for 6.5-m TAO telescope", Proc. SPIE, 8446, 84466P (2012)

[16] Kamizuka, T., Miyata, T., Sako, S., Ohsawa, R., Asano, K., Uchiyama, M., Okada, K., Uchiyama M., Nakamura, T., Sakon, I., Onaka, T., Kataza, H., Aoki, T., Doi, M., Kato, N. M., Kawara, K., Kitagawa, Y., Kohno, K., Konishi, M., Koshida, S., Minezaki, T., Morokuma, T., Motohara, K., Soyano, T., Takahashi, H., Tamura, Y., Tanabé, T., Tanaka, M., Tarusawa, K., Tateuchi, K., Todo, S., and Yoshii, Y., "Revised specifications and current development status of MIMIZUKU: the mid-infrared instrument for the TAO 6.5-m telescope", Proc. SPIE, 9147, 91473C (2014)

[17] Kamizuka, T., Miyata, T., Sako, S., Ohsawa, R., Okada, K., Uchiyama M. S., Mori, K., Yamaguchi, J., Asano, K., Uchiyama, M., Sakon, I., Onaka, T., Kataza, H., Hasegawa, S., Usui, F., Takato, N., Aoki, T., Doi, M., Kato, N. M., Kitagawa, Y., Kobayakawa, Y., Kohno, K., Konishi, M., Minezaki, T., Morokuma, T., Motohara, K., Ohashi, H., Soyano, T., Takahashi, H., Tamura, Y., Tanabé, T., Tanaka, M., Tarusawa, K., Terao, Y., and Yoshii, Y., "Development status of the mid-infrared two-field camera and spectrograph MIMIZUKU for the TAO 6.5-m Telescope", Proc. SPIE, 9908, 99083W (2016)

[18] Uchiyama, M. S., Miyata, T., Kamizuka, T., Sako, S., Ohsawa, R., Okada, K., Mori, K., Yamaguchi, J., Asano, K., and Uchiyama, M., "Development of an optical device (Field Stacker) for achieving accurate photometry in ground-based mid-infrared observations", Proc. SPIE, 9912, 99125N (2016)

[19] Okada, K., Sako, S., Miyata, T., Kamizuka, T., Ohsawa, R., Uchiyama, M. S., Mori, K., Yamaguchi, J., Asano, K., and Uchiyama, M., "Array controller system with cryogenic pre-amplifiers for MIMIZUKU", Proc. SPIE, 9915, 991527 (2016)

[20] Kamizuka, T., Uchiyama, M. S., Yamaguchi, J., Mori, T., Ohsawa, R., Yoshida, Y., Sako, S., Miyata, T., Asano, K., Uchiyama, M., Sakon, I., Onaka, T., Kataza, H., Aoki, T., Doi, M., Hatsukade, B., Kato, N. M., Kohno, K., Konishi, M., Minezaki, T., Morokuma, T., Motohara, K., Soyano, T., Takahashi, H., Tanabé, T., Tanaka, M., Tarusawa, K., Terao, Y., Ohashi, Y., Kono, Yukihiro, Kushibiki, K., and Yoshii, Y., "Laboratory performance evaluation of the mid-infrared camera and spectrograph MIMIZUKU for the TAO 6.5-m telescope", in this conference

[21] Uchiyama, M. S., Miyata, T., Ohsawa, R., Kamizuka, T., Sako, S., Mori, T., Yamaguchi, J., and Yoshida, Y., "Photometric error in mid-infrared observations at the TAO site caused by short-term variation of atmospheric water vapor", in this conference

[22] Mori, T., Miyata, T., Ohsawa, R., Kamizuka, T., Sako, S., Uchiyama, M. S., Yamaguchi, J., and Yoshida, Y., "Performance of the reflective optics of MIMIZUKU at cryogenic temperature", in this conference

[23] Rukde, S. Vanzi, L., C. Schwab, et al., in prep. 
[24] Takahashi, H., Aoki, T., Doi, M., Handa, T., Kamizuka, T., Kato, M. N., Kawara, K., Kohno, K., Konishi, M., Koshida, S., Minezaki, T., Miyata, T., Morokuma, T., Motohara, K., Sako, S., Soyano, T., Tamura, Y., Tanabe, T., Tanaka, M., Tarusawa, K., and Yoshii, Y., "Design of mirror coating facility for The University of Tokyo Atacama Observatory 6.5-m telescop", Proc. of SPIE, vol.9145, 91454N (2014)

[25] Takahashi, H., Yoshii, Y, Doi, M., Kohnoa, K., Miyata, M., Motohara, K., Tanaka, M., Minezaki, T., Morokuma, T., Sako, S., Tamura, Y., Tanabe, T., Konishi, M., Kamizuka, T., Katoa, N., Aoki, T., Soyano, T., and Tarusawa, K., "The University of Tokyo Atacama Observatory $6.5 \mathrm{~m}$ Telescope: design of mirror coating system and its performances", Proc. of SPIE, vol.9906, 99064Q (2016) 